\title{
Editorial
}

\section{Sentinel Node Biopsy Without Scars}

\author{
Does Natural Orifice Transluminal Endoscopic Surgery Herald a New Era for Early GI Cancer?
}

\author{
Hiroya Takeuchi, MD, PhD and Yuko Kitagawa, MD, PhD, FACS
}

Department of Surgery, Keio University School of Medicine, 35 Shinanomachi, Shinjuku-ku, Tokyo 160-8582, Japan

Histopathological status of regional lymph nodes is one of the most significant predictors of recurrence and overall survival for most clinical stage I/II gastrointestinal (GI) cancer, and is often used to justify stratification of patients for adjuvant therapy. More accurate and efficient diagnosis of lymphatic spread and prognostic information can be obtained from a small number of lymph nodes, by intraoperative sentinel node (SN) mapping and sentinel lymphadenectomy. SN navigation surgery is a novel method of minimally invasive surgery which avoids unnecessary lymph node dissection, following diagnosis of nodal metastasis targeting SN.

The clinical applications of SN mapping for GI cancer have been controversial. However, singleinstitutional results, including ours, of SN mapping for early colorectal cancer or gastric cancer are increasingly being considered acceptable in terms of detection rate and accuracy to determine regional lymph node status. ${ }^{1-3}$ Developing a novel laparoscopic minimally invasive colectomy or gastrectomy based on intraoperative SN mapping has been attempted.

Recently the philosophy of surgery has been dramatically revolutionized by the appearance of a new technique referred to as natural orifice transluminal endoscopic surgery (NOTES), because the transluminal approach can yield patient benefit by avoiding any scar on the body surface. ${ }^{4,5}$ NOTES might be figuratively regarded as a second coming of the laparoscopic surgery of the 1990s, and currently there

Published online July 26, 2008.

Address correspondence and reprint requests to: Hiroya Takeuchi, MD, PhD; E-mail: hiroyatakeuchi@aol.com

Published by Springer Science+Business Media, LLC $\odot 2008$ The Society of Surgical Oncology, Inc. has been drastic acceleration in the exploration of new techniques.

The current article by Cahill et al. ${ }^{6}$ reports on lymphatic mapping and SN biopsy by transgastric NOTES in the porcine model. The authors describe that lymphatic mapping and SN biopsy in the colonic mesentery were easily performed with the transgastric NOTES technique, and concluded that SN biopsy is indeed feasible without abdominal incision. The article may represent a valuable signpost for the future direction of SN biopsy for early GI cancer. Combination of endoscopic mucosal resection (EMR) with SN biopsy by NOTES might be an ideal minimally invasive surgical strategy for $\mathrm{cN} 0$ early GI cancer. If the $\mathrm{SN}$ is reliably recognizable via transluminal access and is negative for cancer metastasis, unnecessary radical lymph node dissection with skin incisions could be avoided.

NOTES might herald a new era in surgery; however, there are many technical and ethical issues still to be solved. In fact, the authors already reported the first successful NOTES cholecystectomy in a human being in $2007,{ }^{7}$ and they also mentioned that "we witnessed the introduction of NOTES into clinical practice with mixed feelings of excitement and caution." Even now most endoscopic devices have limitations with regard to surgical procedures such as tissue resection, traction, or suturing. Other issues also remain as follows: Which organ provides the most suitable access route for NOTES? How should the access site be closed? How can we prevent intraperitoneal infection? Should the operators be surgeons or gastroenterologists or both? Several research groups in the USA and Europe have sequentially reported their advances, mainly in animal models. 
Several problems for future clinical applications of SN biopsy by NOTES also remain. Although blue dye is useful for visualizing the lymphatic vessels and $\mathrm{SN}$, it may be difficult for NOTES to visualize the dye due to fast transit and blind sites in dense fat, particularly in obese patients. Radioactive colloid such as technetium-99m-labeled tin colloid may be useful to identify the SN using the NOTES approach. However intraoperative gamma probing and a special gamma detector introducible endoscopically to locate the radioactive SN should be developed for NOTES as soon as possible Furthermore, a well-designed feasibility study of SN mapping by NOTES for early GI cancer should be considered as the next step, because SN mapping has to be extremely accurate as a diagnostic tool for identification of clinically undetectable lymph node metastasis.

To date NOTES has not yet been guaranteed as a safe clinical procedure, and it might need several years for the development of new endoscopes and related devices. However we remember the worldwide explosion of laparoscopic surgery in the 1980s and 1990s. Further exploration may permit innovations of surgical oncology for early GI cancer by SN mapping via NOTES.

\section{REFERENCES}

1. Saha S, Dan AG, Viehl CT, et al. Sentinel lymph node mapping in colon and rectal cancer: its impact on staging, limitations, and pitfalls. Cancer Treat Res 2005; 127:105-22.

2. Bilchik AJ, Saha S, Wiese D, et al. Molecular staging of early colon cancer on the basis of sentinel node analysis: a multicenter phase II trial. J Clin Oncol 2001; 19:1128-36.

3. Kitagawa Y, Fujii H, Mukai M, et al. The role of the sentinel lymph node in gastrointestinal cancer. Surg Clin North Am 2000; 80:1799-809.

4. Kalloo AN, Singh VK, Jagannath SB, et al. Flexible transgastric peritoneoscopy: a novel approach to diagnostic and therapeutic interventions in the peritoneal cavity. Gastrointest Endosc 2004; 60:114-7.

5. Allori AC, Leitman IM, Heitman E. Natural orifice transluminal endoscopic surgery. Arch Surg 2008; 243:333-4.

6. Cahill R, Perretta S, Leroy J, et al. Lymphatic mapping and sentinel node biopsy in the colonic mesentery by natural orifice transluminal endoscopic surgery (NOTES). Ann Surg Oncol (in press).

7. Marescaux J, Dallemagne B, Perretta S, et al. Surgery without scars. Report of transluminal cholecystectomy in a human being. Arch Surg 2007; 142:823-7. 